\title{
Driving Sustainably - The Influence of IoT-based Eco-Feedback on Driving Behavior
}

\author{
Alexander Bätz \\ FIM Research Center, University of Augsburg \\ alexander.baetz@fim-rc.de \\ Henner Gimpel \\ FIM Research Center, University of Augsburg \\ Project Group Business \& Information Systems \\ Engineering of the Fraunhofer FIT \\ henner.gimpel@fim-rc.de
}

\author{
Sebastian Heger \\ FIM Research Center, University of Augsburg \\ Project Group Business \& Information Systems \\ Engineering of the Fraunhofer FIT \\ sebastian.heger@fim-rc.de \\ Moritz Wöhl \\ FIM Research Center, University of Augsburg \\ moritz.woehl@fim-rc.de
}

\begin{abstract}
One starting point to reduce harmful greenhouse gas emissions is driving behavior. Previous studies have already shown that eco-feedback leads to reduced fuel consumption. However, less has been done to investigate how driving behavior is affected by ecofeedback. Yet, understanding driving behavior is important to target personalized recommendations towards reduced fuel consumption. In this paper, we investigate a real-world data set from an IoT-based smart vehicle service. We first extract seven distinct factors that characterize driving behavior from data of 5,676 users. Second, we derive initial hypotheses on how eco-feedback may affect these factors. Third, we test these hypotheses with data of another 495 users receiving eco-feedback. Results suggest that ecofeedback, for instance, reduces hard acceleration maneuvers while interestingly speed is not affected. Our contribution extends the understanding of measuring driving behavior using IoT-based data. Furthermore, we contribute to a better understanding of the effect of eco-feedback on driving behavior.
\end{abstract}

\section{Introduction}

Rising emissions of greenhouse gases (GHG) such as carbon dioxide are accelerating climate change. As things stand, the transportation sector produces $23 \%$ of worldwide GHG emissions [4], a substantial proportion of which is produced by road traffic. A reduction in GHG may be achieved rather quickly via changes in the behavior of drivers [2]. Among others, [7] and [16] outline that, in addition to car characteristics, ecodriving behaviors - such as the maintenance of steady speed - reduce fuel consumption. Thus, a change in driving behavior has the potential to decrease fuel consumption and GHG emissions to increase the sustainability of road travel.

One appropriate tool to reduce fuel consumption is the application of eco-feedback. Various research projects demonstrated a reduction of fuel consumption on average between $1 \%$ and $7 \%$ when providing ecofeedback to the driver [e.g., 3, 6, 25, 27]. This means that eco-feedback must have an effect on driving behavior as driving behavior has a direct impact on fuel consumption [7, 16]. Some studies already considered how separate driving variables change throughout the application of eco-feedback. For instance, [19] analyze the effect of eco-feedback on fuel consumption, as well as, acceleration, deceleration, and average speed. However, research indicates that eco-driving strategies could lead to much higher fuel savings between 5-30\% [26] as compared to the fuel savings in eco-feedback studies. Hence, it is important to understand how driving behavior changes while providing eco-feedback to further develop advanced user assistance systems (AUAS) [18] that encourage and enable drivers to adopt eco-friendly driving strategies [24]. Yet, to date we lack knowledge on how driving behavior changes due to eco-feedback. A prime reason is the traditional lack of data. Average fuel consumption over longer distances and time spans can be measured rather easily. However, until recently it was practically impossible to obtain detailed data on driving maneuvers on scale.

Nowadays, cars' built-in sensors and the standardized OBD-II interface allow access to rich data. Internet-of-Things-(IoT-)based smart vehicle services (SVS) collect comprehensive and detailed data on vehicle activities and driving behavior, such as acceleration, speed, and revolutions per minute (RPM). 
From this data, we can expect a better understanding of the effect of eco-feedback on driving behavior. Crucially, the data reflects authentic and unbiased realworld driving behavior rather than data biased by retrospective self-assessments or behavioral change due to the awareness of taking part in a research project.

The purpose of our paper is to gain a better understanding of the effect of eco-feedback on driving behavior by means of IoT-data. For this reason, our research method consists of five steps. First, real-world driving-data was collected over ten weeks from 5,676 users of an IoT-based SVS. The users were selected randomly from the service's customers. We split the data into two halves and conduct an exploratory factor analysis (EFA) on the first half to derive factors characterizing driving behavior (step 2). Subsequently, we validated factors for driving behavior by means of a confirmatory factor analysis (CFA) on the second half (step 3). Thus, each analysis uses data from 2,838 users. Next, we developed theoretically deduced hypotheses on the effect of eco-feedback on our factors of driving behavior (step 4). To test these, we collected real-world driving data from another 495 users who are both customers of the IoT-based service and customers of an insurance company and therefore qualified for the eco-feedback feature newly introduced by the two companies together. After the first four weeks, the ecofeedback was launched in order to provide ecofeedback to the user group via the use of a mobile app (an integral part of the IoT-based service). Subsequently, we recorded the driving data for another six weeks. Using hypothesis tests, we derive first insights about the effect of eco-feedback on driving behavior (step 5).

Therefore, the remainder of this paper is structured as follows: Section 2 discusses the theoretical background. Section 3 elaborates on our methodology, data set, and the study design. Section 4 presents the results of the factor analyses. Subsequently, we derive initial hypotheses and gain first insights. Section 5 discusses our findings.

\section{Theoretical background}

\section{1. (Eco-)driving behavior}

Driving behavior has two fundamental aspects. The first of these is strategic driving behavior, also referred to as travel behavior. It includes, among other things, the chosen route and trip goals such as minimizing time or costs but also the choice of transportation mode $[3,20]$. The second aspect of driving behavior is execution-related. It encompasses both tactical and operational driving behavior, and how driver attitudes are reflected in either calm or aggressive driving behavior [20, 21]. Studies have found that calm driving behavior is characterized by a low gear-shifting frequency, slow acceleration, and driving speeds not exceeding the legal limit. Aggressive driving,on the other hand, involves a higher tendency to shift gears, hard acceleration, and speeds above the legal speed limit [21]. The existing literature investigates the effect of these fundamental aspects of driving behavior on fuel consumption. [7] conducted a factor analysis based on 62 driving parameters to enlarge the rather general aspects of driving behavior. According to her results, 16 different and independent factors describe driving behavior. Of these, moderate and hard acceleration, a strong speed oscillation, many stops during a trip and late gear changes from gear 2 to 3 increase fuel consumption. On the other side, deceleration, driving speed between 50 and $90 \mathrm{~km} / \mathrm{h}$, moderate engine speed at gears 2 and 3 as well as low engine speed at gears 4 and 5 decrease fuel consumption. These results are also consistent with the results of [16]. The driven distance, hard acceleration, and a higher average speed increase fuel consumption per kilometer driven. Moreover, results show a higher fuel consumption for hard deceleration and if one considers the number of stops and the idle time during a trip separately [16].

Hence, it is important to achieve eco-driving behavior to reduce fuel consumption and thus contribute to the fight against rising GHG emissions.

\subsection{The influence of feedback on fuel consumption}

Previous research suggests that a person's behavior can be improved by providing feedback [8]. Feedback is a "communications process in which some sender [...] conveys a message to a recipient [...] [that] comprises information about the recipient." [13]. According to the feedback intervention theory, this information enables the creation of a gap between a person's behavior and some standard or individual goal. Resulting in a person's desire to reduce this gap. To close this gap, feedback can vary from a high-level to a detailed one [14]. First, feedback can draw a person's attention to a specific problem and thus encourage to consider the ways in which a person's behavior may contribute [8]. In this case, feedback rather shows the high-level consequences of one's actions [14]. One example is the environmental damage caused by high fuel consumption. Second, feedback can raise people's awareness of the relevance of their own behavior. Likewise, it can increase people's understandings of the consequences of behavioral change. In this way, feedback makes clear 
the links between the actions of individuals and the problem at hand, for example by explaining the increases and decreases in fuel consumption that result from specific actions [8]. In this form, feedback offers a detailed description of the necessary actions to change a person's behavior [14]. Lastly, feedback can provide additional motivation for behavioral change [8]. For example, feedback may foster a competitive environment, which provokes an improvement in a person's behavior due to the gap between one's own behavior and that of others [14].

Various studies have already investigated the effect of eco-feedback on fuel consumption. Eco-feedback is likely to improve rather strategic driving behavior such as reducing car usage and therefore annual mileage at all. For instance, [11] find a positive effect when providing eco-feedback on environmental and financial savings $\left(\mathrm{CO}_{2}\right.$ and money) to a group of students while they do not use their cars.

Also, eco-feedback improves rather executionrelated driving behavior and, therefore, contributes to decreasing fuel consumption per driven kilometer. As early as 1989, feedback along with other information as well as task assignment and control were considered as influencing factors to reduce one's energy consumption [25]. As one of the first researches, the study mainly concentrated on mail-van drivers of the Netherlands Postal and Telecommunication Services. To provide these drivers with feedback about their fuel consumption, they used a simple notice on a bulletin board in the drivers' canteen. According to their results, fuel savings of $7.3 \%$ were achieved [25]. However, the reduction in fuel consumption could not be attributed exclusively to feedback. To address this issue, [29] conducted an experiment in a driving simulator. Within the simulated environment, the system provided the subjects with their individual fuel consumption based on their actual driving behavior. Their results also show a $7 \%$ reduction in fuel consumption [29].

With the spread of digital technologies, also the presentation of the feedback has changed. Technological progress allows to better investigate the influence of feedback directly in real-world applications. To collect the necessary data, the OBD-II interface of the car may be accessed during a trip. The feedback is then displayed using an eco-driving device [3]. The results for the 23 selected participants of the study [3] show a decrease of $6 \%$ while driving in the city and $1 \%$ for highway driving. A similar approach was conducted by [27] using a smartphone application to present feedback to 50 corporate drivers. They find an improvement in fuel efficiency of $3 \%$ and explain the smaller effect compared to previous studies with the real-world scenario in which the data was collected as well as with the absence of any incentive to the drivers [27]. [15] support the results of [27] as they find a similar reduction in fuel consumption in a realworld scenario. However, all of the participants in [15] were motivated by monetary incentives. Since the results of [27] and [15] are quite similar but differ in the provision of incentives, incentives are not the main reason for the smaller impact of feedback, which is in line with the findings of [6].

\subsection{The influence of feedback on driving behavior}

Feedback must have an effect on one's driving behavior that leads to a decrease in fuel consumption $[7,16]$. However, little has been done to understand the effect of eco-feedback on driving behavior.

Given the reduction of fuel consumption upon ecofeedback, it can be assumed that drivers change some aspects of their driving behavior based on ecofeedback. However, it seems that optimal driving strategies can save more fuel than the results of the eco-feedback studies show [cf. 3, 26, 27].

An explanation is that the effect of eco-feedback does not sufficiently address all factors of driving behavior. For instance, [19] show that eco-feedback on fuel consumption and $\mathrm{CO}_{2}$ emissions reduces fast driving and favors slow driving. [28] provide rather behavior-specific feedback on uneconomical power demands of the engine. Their aim is to reduce acceleration and early upshifting. Results show successful improvements in relation to these two driving factors. [11] provide feedback on car usage and achieve significant reductions in annual mileage.

Summarizing, eco-driving has an impact on fuel consumption and thus GHG emissions. Numerous scientific studies have shown that feedback is a possible approach to motivating ecological driving behavior and thereby saving fuel. However, there is a lack of research investigating which factors from data of an IoT-based SVS describe driving behavior and how these are affected by eco-feedback.

\section{Method}

\subsection{Design of the eco-feedback}

In addition to our study design (as stated in the introduction), the SVS provides eco-feedback to 495 drivers, delivered via a mobile app. At the end of each trip, an eco-score between 0 and $100 \%$ is calculated and sent to the participant's mobile device. The total score is composed of four separate scores, each ranging from 0 to 25 , together adding up to a maximum of 100 . 
The four scores reflect: average acceleration in comparison to other users of the IoT-service; average deceleration in comparison to other users of the IoTservice; trip length (short journeys $(<5 \mathrm{~km})$ by car are classified as less environmentally friendly); and the proportion of the trip driven at speeds in excess of $130 \mathrm{~km} / \mathrm{h}$ (which is a reasonable threshold in Germany, as there is no general speed limit). Participants receive a push notification at the end of each trip (Figure 1, bottom) with the score and some details about length, duration, speed, and acceleration of the completed trip. In addition, participants are able to view their latest score, as well as the average score, when they open the mobile app (Figure 1, top).

\subsection{Variables in the data sets}

The service records driving data via the on-board diagnostic interface (OBD-II). Recording data enables the service to offer its users insights into their cars (e.g., battery level, location of the car), and locationbased services (e.g. discounts at selected petrol stations), via their mobile devices. Since our data was recorded before the inception of this study, users were not aware of their inclusion and thus our results are unbiased. To preserve privacy, and in accordance with the service's privacy policy, we do not know the identities of users, nor do we have access to any location data. All included users have agreed to the anonymous use of their data in advance by accepting the privacy policy of the IoT-based SVS. From the SVS, we draw the following available data for each short stretch of way of each trip: speed (in $\mathrm{km} / \mathrm{h}$ ), acceleration, deceleration (both in $\mathrm{g}$ ), revolutions per minute, driving time, standing time (both in seconds), driven distance (in meter), throttle (in degree), and engine coolant temperature (in ${ }^{\circ} \mathrm{C}$ ).

Unfortunately, the service does not record which gears are engaged. Instead, we use the RPM as an approximation of the gear-shifting behavior. The variables for acceleration and deceleration exhibit outliers. Some observations exceed or fall below plausible values and, thus, are capped. We computed the $99 \%$-quantile for acceleration and deceleration and capped observations to eliminate outliers. As the sensing device delivers data continuously, there was no need to handle missing data. Following this data cleansing, we aggregated the single values of each variable for a whole week in order to enable a meaningful comparison of a person's driving behavior in regular time intervals. Thus, the aggregation reduces inaccuracies, which may result for instance from differences in driving behavior on working and leisure days, as well as effects of chance. The aggregation is done by computing several of the following summary statistics: minimum, maximum, sum, average, variance, and $95 \%$-quantile.

\section{Results}

\subsection{Identification of factors characterizing driving behavior}

To assess the effect of feedback on driving behavior, we need to clarify the measurable factors of driving behavior in our IoT data. Consequently, we conduct an EFA on the driving data of 2,838 drivers in $\mathrm{R}$ [22]. In total, the data comprises of 36 variables describing the driving behavior (different weekly aggregations of the aforementioned variables). 35 variables fulfill the Kaiser-Meyer-Olkin criterion with a measure of sampling adequacy (MSA) above 0.5 and thus are further analyzed. Overall the MSA is 0.79 which indicates the data is suitable for conducting an EFA. Same applies to Bartlett's test of homogeneity which is significant ( $\mathrm{p}$-value $<0.001)$. A parallel analysis [12] initially suggests ten factors. After oblimin rotation, we excluded items with a major loading below 0.4. As a result, one variable (average engine coolant temperature) is dropped. Furthermore, no item substantially loads on the tenth factor which is then removed from the analysis. Rerunning the factor analysis with nine factors results in the loadings displayed in Table 1. Factor nine is a subset of factor four and due to the higher loadings and eigenvalue of factor four ignored. The result of the EFA are eight factors which describe aspects of strategic and operational driving behavior.

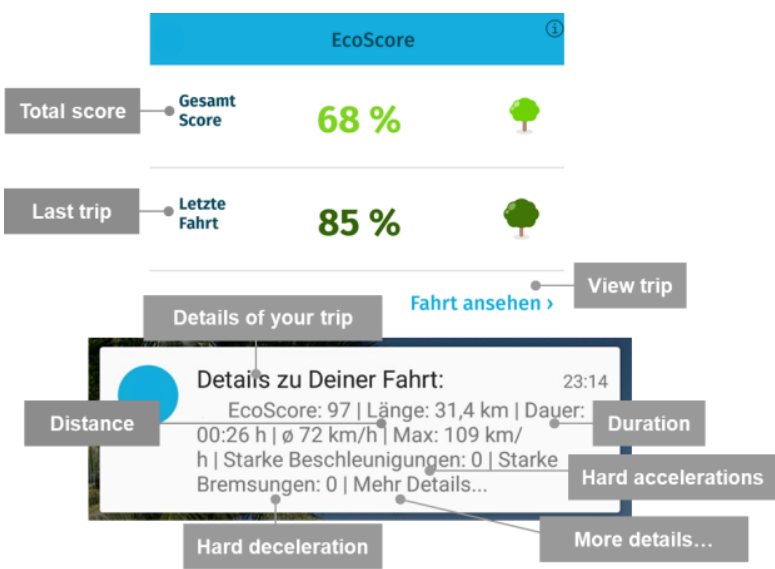

Figure 1. Visualization of the eco-score in the app (top) and as push notification (bottom); (translated)

To ensure our results will be useful, we need to be able to interpret our factors. If a single factor cannot be interpreted, the usefulness of the results is limited. In the following, we describe the eight factors identified, which serve as our understanding of driving behavior. 
The first factor can be interpreted as "acceleration behavior". It includes the average, maximum, 95\%quantile, and variance of acceleration and deceleration. The second factor is the "total driving per week" composed of the total distance, time in the car, and the number of trips per week. The third factor can be interpreted as the "speed behavior" encompassing the average and maximum speed as well as the variance of speed. The fourth factor is related to the first factor "acceleration behavior" with the difference that it covers the number and frequency of extreme events of acceleration or deceleration. The factor can be interpreted as "extreme acceleration behavior". The thresholds for those events are defined by the SVS provider. The fifth factor reflects the "average driving per week" in the sense of average trip distance and average trip duration. The sixth factor can be interpreted as "minimum driving per week" which reflects the shortest trip distance and duration per week. The seventh factor is "RPM behavior" consisting of the average, maximum, and variation of the RPM of the engine. Last is the "throttle behavior", reflected by average, maximum, and variation of the throttle position.

Most factors include at least one loading greater than 0.7 but one, the throttle factor, has two indicators slightly missing that threshold. These eight factors cover $66 \%$ of the variance in the data (cf. Table 2). Cronbach's alpha for our factors is mostly excellent. Only the throttle factor does not reach 0.7 and, thus, is removed for further analysis.

For some factors like RPM, it might appear straightforward that average, maximum and variance are highly correlated, although technically this is not necessarily the case but a result. For other factors, the structure is less straightforward; examples are the combination of acceleration and deceleration in the first factor but the separation of factor one and four.

Overall, our factor model seems to be consistent with the factors identified by [7]. However, due to different availability of data, our factor model encompasses three factors of strategic driving behavior (total, average and minimum driving per week) and four factors related to operational driving behavior (acceleration, extreme acceleration, speed, and RPM behavior), while [7]'s factor model solely consists of factors related to operational driving behavior, therefore our factor model is more comprehensive.

\subsection{Validation of factors characterizing driving behavior}

Following the development of our model for driving behavior, we validate our factors for driving behavior by applying a CFA on an independent data set from the SVS provider. The data set results from a new set of 2,838 users. The CFA shows good values $(>0.7)$ in terms of Cronbach's Alpha $(\mathrm{C} \alpha)$ for all seven factors. Values of 0.6 regarding the composite reliability (CR) and 0.5 for average variance extracted (AVE) can be seen as good measurement quality [1]. All seven factors did meet these thresholds.

We check the discriminant validity for the seven factors by using the Fornell-Larcker criterion that a factor's AVE should be higher than its squared correlation with every other factor [9]. Table 3 summarizes the results. We can assume discriminant validity for all seven factors of driving behavior.

In summary, we can state that we have found based on the data provided by a SVS provider - a valid factor structure for assessing driving behavior.

\subsection{Developing hypotheses for driving behavior affected by feedback}

Having identified seven factors which describe driving behavior on the basis of our IoT-data set, in the following, we introduce hypotheses how the ecofeedback could affect driving behavior.

[19] find evidence that both acceleration and deceleration are likely to improve if drivers receive eco-feedback on their driving behavior. Especially forceful acceleration increases fuel consumption and is therefore likely to be decreased by knowledgeable and eco-conscious drivers $[7,16]$. Deceleration, in contrast, has no direct impact on fuel consumption but causes acceleration back up to speed [16]. Furthermore, the eco-feedback of the IoT-based SVS punishes hard acceleration and deceleration by a deterioration of the eco-score. The driver, in addition, receives the number of hard acceleration and deceleration events in the trip summary. Thus, we assume that acceleration and deceleration will improve when providing feedback to the driver, which are reflected in two factors.

H1(a) Providing eco-feedback decreases the factor "acceleration behavior" (b) Providing eco-feedback decreases the factor "extreme acceleration behavior"

In addition to changes in acceleration and deceleration behavior, [19] also find evidence that ecofeedback reduces speed behavior. Speed impacts fuel consumption resulting in fuel savings especially when driving at moderate speed (between 50 and $70 \mathrm{~km} / \mathrm{h}$ ) [7, 16]. Accordingly, we assume that our feedback has a reducing effect on speed.

H2: Providing eco-feedback decreases the factor "speed behavior"

Another factor of driving behavior from our factor analysis is the RPM which relates to speed and gears engaged. Eco-friendly driving requires early upshifts and therefore low RPM [24]. However, the eco- 
feedback of the IoT-based SVS does not address either the RPM or the shifting behavior. Total driving per week is significantly influenced by feedback which supports H5. However, the magnitude of the effect seems to be negligible. Nevertheless, we observe that, for example, the number of trips per week decreases by 0.54 trips per user of the IoT-based SVS.

Table 1. Rotated factor matrix from EFA (with loadings $\geq 0.4$ )

Note: Factors can be named as follows: (1) acceleration behavior, (2) total driving per week, (3) speed behavior, (4) extreme acceleration behavior, (5) average driving per week, (6) minimum driving per week, (7) RPM behavior, (8) throttle behavior, (9) disregarded as subset of factor (4)

\begin{tabular}{|c|c|c|c|c|c|c|c|c|c|}
\hline \multirow[b]{2}{*}{ Original Parameter } & \multicolumn{9}{|c|}{ Factor } \\
\hline & 1 & 2 & 3 & 4 & 5 & 6 & 7 & 8 & 9 \\
\hline Average Acceleration & 0.73 & & & & & & & & \\
\hline Maximum Acceleration & 0.84 & & & & & & & & \\
\hline 95\%-Quantile Acceleration & 0.76 & & & & & & & & \\
\hline Variance Acceleration & 0.75 & & & & & & & & \\
\hline Average Deceleration & 0.79 & & & & & & & & \\
\hline Maximum Deceleration & 0.89 & & & & & & & & \\
\hline 95\%-Quantile Deceleration & 0.89 & & & & & & & & \\
\hline Variance Deceleration & 0.89 & & & & & & & & \\
\hline \# Trips & & 0.85 & & & -0.43 & & & & \\
\hline Sum Distance & & 0.64 & & & & & & & \\
\hline Sum Duration & & 0.91 & & & & & & & \\
\hline Sum Driving Duration & & 0.83 & & & & & & & \\
\hline Sum Standing Duration & & 0.93 & & & & & & & \\
\hline \# Long Stops (>3m) & & 0.69 & & & & & & & \\
\hline \# Stops & & 0.51 & & & & & & & \\
\hline Average Speed of Trip Averages & & & 0.94 & & & & & & \\
\hline Average Speed & & & 0.64 & & & & & & \\
\hline Average Speed when Driving & & & 0.93 & & & & & & \\
\hline Maximum Speed & & & 0.70 & & & & & & \\
\hline Variance Speed & & & 0.70 & & & & & & \\
\hline \# Hard Accelerations & & & & 0.75 & & & & & \\
\hline Hard Accelerations per KM & & & & 0.85 & & & & & \\
\hline \# Hard Decelerations & & & & 0.47 & & & & & 0.45 \\
\hline Hard Decelerations per KM & & & & 0.53 & & & & & 0.47 \\
\hline Average Trip Distance & & & & & 0.70 & & & & \\
\hline Average Trip Duration & & & & & 0.86 & & & & \\
\hline Minimum Trip Distance & & & & & & 0.90 & & & \\
\hline Minimum Trip Duration & & & & & & 0.92 & & & \\
\hline Average RPM & & & & & & & 0.66 & & \\
\hline Maximum RPM & & & & & & & 0.85 & & \\
\hline Variance RPM & & & & & & & 0.68 & & \\
\hline Average Throttle & & & & & & & & 0.68 & \\
\hline Maximum Throttle & & & & & & & & 0.67 & \\
\hline Variance Throttle & & & & & & & & 0.55 & \\
\hline
\end{tabular}


Table 2. Descriptive statistics of factors in EFA

Note: Factor numbering as in Table 1, Cronbach's Alpha should be $>0.7$

\begin{tabular}{|c|c|c|c|c|c|c|c|c|c|c|c|}
\hline \multirow{2}{*}{\multicolumn{2}{|c|}{ Statistics }} & \multicolumn{10}{|c|}{ Factor } \\
\hline & & 1 & 2 & 3 & \multicolumn{2}{|l|}{4} & 5 & 6 & 7 & 8 & \multirow{2}{*}{$\frac{9}{0.02}$} \\
\hline \multicolumn{2}{|c|}{ Proportion Variance } & 0.16 & 0.13 & 0.11 & 0 . & & 0.05 & 0.05 & 0.05 & 0.04 & \\
\hline \multicolumn{2}{|c|}{ Cumulative Variance } & 0.16 & 0.29 & 0.41 & 0 . & & 0.52 & 0.57 & 0.62 & 0.66 & 0.68 \\
\hline \multicolumn{2}{|c|}{ Cronbach's Alpha } & 0.96 & 0.91 & 0.91 & 0 . & & 0.97 & 0.91 & 0.75 & \multicolumn{2}{|l|}{0.51} \\
\hline \multicolumn{12}{|c|}{$\begin{array}{l}\text { Table 3. Descriptive statistics of CFA } \\
\text { Note: Factor numbering as in Table } 1 \\
\text { n, diagonal elements represent } A V E \text { and off-diagonal elements correlations } \\
\text { if: Cronbach's Alpha }>0.7 \text {, Composite-reliability }>0.6, A V E>0.5 \text {, and Fornell- } \\
\text { met, i.e., AVE is higher than the squared correlation with any other factor }\end{array}$} \\
\hline & & & & & \multicolumn{7}{|c|}{ Fornell-Larcker criterion } \\
\hline \multicolumn{3}{|c|}{ Factor } & $\mathrm{C} \alpha$ & $\mathrm{CR}$ & (1) & (4) & (3) & (7) & (6) & (5) & $(2)$ \\
\hline \multirow{2}{*}{\multicolumn{3}{|c|}{$\begin{array}{l}\text { (1) acceleration behavior } \\
\text { (4) extreme acceleration } \\
\text { behavior }\end{array}$}} & 0.96 & 0.96 & 0.77 & & & & & & \\
\hline & & & 0.79 & 0.90 & 0.47 & 0.82 & & & & & \\
\hline \multicolumn{3}{|c|}{ (3) speed behavior } & 0.91 & 0.84 & -0.14 & -0.04 & $\mathbf{0 . 5 3}$ & & & & \\
\hline \multicolumn{3}{|c|}{ (7) RPM behavior } & 0.91 & 0.92 & 0.11 & 0.12 & 0.24 & 0.79 & & & \\
\hline \multicolumn{3}{|c|}{ (6) minimum drivir } & 0.95 & 0.98 & -0.06 & -0.03 & 0.29 & -0.05 & 0.95 & & \\
\hline \multicolumn{3}{|c|}{ (5) average driving per week } & 0.97 & 0.98 & -0.29 & -0.04 & 0.67 & 0.11 & 0.42 & 0.97 & \\
\hline \multicolumn{3}{|c|}{ (2) total driving per week } & 0.90 & 0.93 & -0.12 & 0.11 & 0.18 & 0.13 & -0.15 & 0.37 & 0.74 \\
\hline
\end{tabular}

For this reason, we assume that this factor will not change, although an eco-friendly strategy would require low RPM.

H3: Providing eco-feedback does not affect the factor "RPM behavior"

Eco-feedback can address driving behavior on a rather strategic level as well. [11] uses eco-feedback successfully to reduce the number of rides and therefore the overall mileage. The study reflects to the participants what environmental $\left(\mathrm{CO}_{2}\right)$ and financial savings have been achieved by not using the car.

Eco-feedback from the IoT-based SVS reduces the eco-score when the vehicle is used for short distances, reflecting non-ecological use. Accordingly, we expect that short distances are avoided and, therefore, the remaining trips become longer on average.

H4(a) Providing eco-feedback increases the factor "minimum driving per week" and (b) increases the factor "average driving per week"

The last factor of driving behavior in our study is the factor "total driving per week". We assume that the distance covered decreases because short distances are avoided (see H4a, H4b). In addition, the eco-feedback could create awareness for every trip taken [11]. Thus, we hypothesize that users may leave their car and use alternative means of transport leading to less overall driving per week.

H5: Providing eco-feedback decreases the factor

"total driving per week"

\subsection{Analysis of the effect of eco-feedback}

Finally, to assess the effect eco-feedback has on driving behavior we conduct our analysis on a third data set consisting of 495 drivers. For every driver, we have baseline driving data of four weeks. The ecofeedback was launched during week 5. To ensure the baseline and the treatment phase are not mixed, this week is removed from the data set. Following the launch of the eco-feedback, the data set comprises of six weeks of data per driver. As not every car was used every week, the baseline record per driver is 3.79 weeks and 5.37 weeks for the treatment phase on average. Based on the factors we identified, we calculate the factors of driving behavior per week perdriver. We assess the effect of whether and in which direction feedback influences driving behavior based on the aggregated driving factors. Consequently, the factor scores per driver are averaged before and after the start of the feedback. First, we check whether the aggregated factor scores are normally distributed to 
choose the appropriate test. Consequently, we perform the Shapiro-Wilk test of normality.

The results suggest that factor scores are not normally distributed across the drivers. As a consequence, we compare the factor scores before and after the launch of the feedback applying the Wilcoxon signed-rank test. For H3 where we assume no effect, we perform a two-sided test. For all other hypotheses, we perform one-sided tests. In addition, we check not only the presence and direction of an effect but also the effect size (Cohen's d). The effect size is calculated by subtracting the mean of the experimental group (here: after application of eco-feedback) from the mean of the control group (here: before the application of the ecofeedback) and dividing the difference by the standard deviation of the data. Thus, a negative value of Cohen's $d$ indicates a decrease in the respective factor due to the display of feedback. A positive value vice versa indicates an increase in the factor score. An absolute value of the effect size of 0.2 is termed a "small effect" [5].The results of the Wilcoxon signedrank test and the effect size are depicted in Table 4.

Our data supports H1a and H1b. We conclude that in terms of acceleration behavior as well as extreme acceleration behavior, feedback does have a desired, significant, and meaningful (small) effect. Drivers seem to accelerate and decelerate more carefully.

In terms of speed and RPM, we find no significant effects of the eco-feedback. Consequently, we reject $\mathrm{H} 2$ whereas $\mathrm{H} 3$ is supported. However, while the average speed slightly increases from $31.51 \mathrm{~km} / \mathrm{h}$ to $31.76 \mathrm{~km} / \mathrm{h}$ the average maximum speed slightly decreases from $135.51 \mathrm{~km} / \mathrm{h}$ to $134.69 \mathrm{~km} / \mathrm{h}$, which appears to be favorable as especially high speeds cause higher fuel consumption.

The factors for minimum and average driving per week are significantly influenced by the application of the eco-feedback in that the minimum and average trip length and duration increase. This supports $\mathrm{H} 4 \mathrm{a}$ and $\mathrm{H} 4 \mathrm{~b}$. It seems as if our assumption was correct that short distances are avoided and thus the average trip becomes longer. Both the minimal trip distance and the average distance per trip increase from $459 \mathrm{~m}$ to $606 \mathrm{~m}$ and $11.24 \mathrm{~km}$ to $12.02 \mathrm{~km}$ respectively. Considering the effect size, the effect is statistically significant but not substantial for both factors.

Table 4. Summary of effect of eco-feedback (FB) on driving behavior Note: ${ }^{* * *} p$-value $<0.1 \%$, ${ }^{* *} p$-value $<1 \%$, ${ }^{*} p$-value $<5 \%$

Factor p-value of Wilcoxon

(1) acceleration behavior signed-rank test effect size Hypothesis Result

(4) extreme acceleration behavior

(3) speed behavior $<0.001 * * *$ $-0.19$ H1a: Decrease through FB Support

(7) RPM behavior $<0.001 * * *$ $-0.18$ H1b: Decrease through FB

Support

(6) minimum driving per

H2: Decrease through FB

Reject

(6) week

0.203

$-0.03$

H3: No effect through FB

Support average driving per week

(2) total driving per week

$0.016 *$

H4a: Increase through FB

Support

\section{Discussion and conclusion}

In this study, we derive seven factors from data of an IoT-based SVS, which describe strategic and operational driving behavior. Furthermore, we investigate how eco-feedback impacts driving behavior. We show which aspects of driving behavior are positively influenced due to the application of ecofeedback in a real-world scenario.

A strength of our study is the large real-world data set exceeding previous studies, arguably leading to more generalizable results. In addition, our data is not biased by the Hawthorne effect [23], as participants did not know their driving behavior was investigated in the context of the eco-feedback. We, thus, can assume the observed effects are due to the intrinsic motivation of the participants and not due to the fact that they were asked to participate in a study. Even if the measured effects are only small or almost negligible, if the lever is big enough these effects still can make an important impact. With regards to climate change, almost any effort is important and even small steps can contribute to making transportation more sustainable.

The theoretical contribution of this paper is a comprehensive factor model explaining driving behavior on a strategic and operational level. In comparison to [7], the variables underlying our factor model do not need additional specific data collection equipment as the respective data can be obtained via 
the OBD-II-interface and, thus, is widely applicable especially as IoT-solutions push into the market for additional driving features. Further, we have shed light on how drivers adjust their driving behavior based on eco-feedback which reflects how drivers expect to drive more sustainably. While we observe the strongest effects with regards to operational driving behavior (acceleration behavior and extreme acceleration behavior), the effects on strategic driving behavior (average, minimum and total driving per week) seem to be smaller and, thus, eco-feedback seems to be less effective with regards to this concern. Conclusively, eco-feedback alone may not be sufficient to grasp the full potential for more eco-friendly driving. For example, AUAS [18] may supplement feedback with other approaches like goal-setting, rewards [10], or personalized recommendations [28] to improve previously unaddressed behavior along the path towards autonomous vehicles.

Besides the theoretical contribution, this work offers managerial implications. Our findings are relevant with regards to designing future feedback systems in the automotive sector. We derive first insights which allow car manufacturers, insurance companies, as well as third-party applications to tailor feedback to make it more effective. Practitioners and researchers may build on our findings to gain a better understanding of how to design AUAS [18] to reduce the emission of greenhouse gases. In doing so, information systems can contribute to a more sustainable lifestyle and help to reduce harmful greenhouse gas emissions. Moreover, lower greenhouse gas emissions result from reduced fuel consumption which leads to lower costs. Thus, feedback may help companies, especially logistics companies, to save money as their daily business consists of many vehicles and drivers. Further, ecofriendly driving tends to go along with safe driving which is the reason why a car insurance company sponsored the development of the eco-feedback functionality in the IoT-service. Hence, the benefits of IoT-based eco-feedback may go beyond the positive effect on environmental sustainability.

Despite the rigor of our study, our findings are subject to some limitations. We provided the ecofeedback only within a mobile app. We could not ensure that all participants regularly checked their ecofeedback or truly received the push notifications. Furthermore, we cannot be certain that the cars involved in the study were driven only by our participants. Thus, the presumed effects of ecofeedback on driving behavior might, in fact, be stronger when the feedback would be more salient in the car. Our data setis limited by the variables that the SVS has chosen to measure and disclose to us. As a consequence, our factor model does lack certain variables which are not measured or disclosed by the SVS provider, like gear-shifting, type of road or the actual fuel consumption. Additional variables could enhance the factor model and further investigate and improve the effectiveness of feedback. Our sample was restricted to customers of the service, which implies a limitation to Germany and possibly a self-selection bias as customers are presumably more interested in vehicles and potentially care about their driving style. We only considered the effect of eco-feedback in the short term as our data set only contains information about the variables for ten weeks in total. Finally, the analysis of the effect of eco-feedback does not include a control group and, thus, might be affected by unmeasured or uncontrolled external conditions, i.e. changes in weather between the period prior and after the launch of the eco-feedback. We are in the process of obtaining data for a control group. Nevertheless, the findings offer promising first insights and provide a starting point for future research.

Based on our factor model, more sophisticated analyses are conceivable, which could consider that, for example, speed or RPM are no linear function in terms of fuel consumption and the effectiveness of feedback. However, not only the analysis could be extended, but also the model itself. Hence, the measurement of additional variables could describe driving behavior in more detail. In addition, future workcould focus on specific groups of drivers, selected on the basis of either similar driving behavior or personal factors. Personal factors could be of relevance in this field as Lewin's equation states that behavior here driving behavior - is a function of the environment (here: among other influences, the provided feedback) and the person, respectively personal factors, which are not investigated in this study [17]. This will allow further investigations into the effects of eco-feedback on specific sub-groups and will, thus, enable more customized and effective feedback in a real-world setting. In addition, driving patterns could be used to evaluate different types of feedback in order to increase impact, as the feedback applied in our study presumably influenced participants with environmental awareness. Finally, future research might investigate whether a person's (operational) driving behavior is unique - like a fingerprint - and, if so, whether it may, for example, be used to prevent insurance fraud.

In summary, we believe that data from IoT-based SVSs offer a promising opportunity to better understand the effect of feedback and to make feedback and AUAS even more efficient. 


\section{References}

[1] Bagozzi, R.P. and Y. Yi, "On the evaluation of structural equation models", Journal of the Academy of Marketing Science, 16(1), 1988, pp. 74-94.

[2] Barkenbus, J.N., "Eco-Driving: An Overlooked Climate Change Initiative", Energy Policy, 38(2), 2010, pp. 762769.

[3] Boriboonsomsin, K., A. Vu, and M. Barth, Eco-Driving: Pilot Evaluation of Driving Behavior Changes Among U.S. Drivers, Riverside: University of California Transportation Center.

[4] https://www.ipcc.ch/report/ar5/wg3/, accessed 6-8-2019.

[5] Coe, R., "It's the effect size, stupid", a paper presented at the British Educational Research Association annual conference, 2002.

[6] Dahlinger, A., V. Tiefenbeck, B. Ryder, B. Gahr, E. Fleisch, and F. Wortmann, "The impact of numerical vs. symbolic eco-driving feedback on fuel consumption - A randomized control field trial", Transportation Research Part D: Transport and Environment, 65, 2018, pp. 375386.

[7] Ericsson, E., "Independent Driving Pattern Factors and their Influence on Fuel-Use and Exhaust Emission Factors", Transportation Research Part D: Transport and Environment, 6(5), 2001, pp. 325-345.

[8] Fischer, C., "Feedback on Household Electricity Consumption: A Tool for Saving Energy?", Energy Efficiency, 1(1), 2008, pp. 79-104.

[9] Fornell, C. and D.F. Larcker, "Evaluating Structural Equation Models with Unobservable Variables and Measurement Error", Journal of Marketing Research, 18(1), 1981, p. 39.

[10] Froehlich, J., L. Findlater, and J. Landay, "The design of eco-feedback technology", in Proceedings of the 28th International Conference on Human Factors in Computing Systems - CHI '10, E. Mynatt, D. Schoner, G. Fitzpatrick, S. Hudson, K. Edwards, and T. Rodden, Editors, Atlanta, Georgia, USA. 2010. ACM Press: New York, New York, USA.

[11] Graham, J., M. Koo, and T.D. Wilson, "Conserving Energy by Inducing People to Drive Less", Journal of Applied Social Psychology, 41(1), 2011, pp. 106-118.

[12] Horn, J.L., "A rationale and test for the number of factors in factor analysis", Psychometrika, 30(2), 1965, pp. 179-185.

[13] Ilgen, D.R., C.D. Fisher, and M.S. Taylor, "Consequences of individual feedback on behavior in organizations", Journal of Applied Psychology, 64(4), 1979, pp. 349-371.

[14] Karlin, B., J.F. Zinger, and R. Ford, "The effects of feedback on energy conservation: A meta-analysis", Psychological Bulletin, 141(6), 2015, pp. 1205-1227.

[15] Kurani, K., Impact of in-car instruments on driver behavior, Presentation at the Eco-Drive Workshop, 2007.
[16] Lárusdóttir, E.B. and G.F. Ulfarsson, "Effect of Driving Behavior and Vehicle Characteristics on Energy Consumption of Road Vehicles Running on Alternative Energy Sources", International Journal of Sustainable Transportation, 9(8), 2014, pp. 592-601.

[17] Lewin, K., Principles of Topological Psychology, Mcgraw-Hill Book Company, New York, NY, 1936.

[18] Maedche, A., S. Morana, S. Schacht, D. Werth, and J. Krumeich, "Advanced User Assistance Systems", Business \& Information Systems Engineering, 58(5), 2016, pp. 367-370.

[19] Martin, E.W., K. Boriboonsomsin, N.D. Chan, N. Williams, S.A. Shaheen, and M. Barth, "Dynamic ecodriving in northern california: A study of survey and vehicle operations data from an ecodriving feedback device", in 92nd Annual Meeting of the Transportation Research Board, Washington D.C. 2013.

[20] Michon, J.A., "A Critical View of Driver Behavior Models: What Do We Know, What Should We Do", in Human Behavior and Traffic Safety, L. Evans and R.C. Schwing, Editors. 1985. Springer US: Boston, MA.

[21] Rafael, M., M. Sanchez, V. Mucino, J. Cervantes, and A. Lozano, "Impact of Driving Styles on Exhaust Emissions and Fuel Economy From a Heavy-Duty Truck: Laboratory tests", International Journal of Heavy Vehicle Systems, 13(1/2), 2006, p. 56.

[22] Revelle, W. and M.W. Revelle, "Package 'psych'".

[23] Rice, B., "The Hawthorne Defect: Persistence of a Flawed Theory", Psychology Today, 16(2), 1982, pp. 7074.

[24] Saboohi, Y. and H. Farzaneh, "Model for developing an eco-driving strategy of a passenger vehicle based on the least fuel consumption", Applied Energy, 86(10), 2009, pp. 1925-1932.

[25] Siero, S., M. Boon, G. Kok, and F. Siero, "Modification of Driving Behavior in a Large Transport Organization: A field experiment", Journal of Applied Psychology, 74(3), 1989, pp. 417-423.

[26] Sivak, M. and B. Schoettle, "Eco-driving: Strategic, tactical, and operational decisions of the driver that influence vehicle fuel economy", Transport Policy, 22, 2012, pp. 96-99.

[27] Tulusan, J., T. Staake, and E. Fleisch, "Providing EcoDriving Feedback to Corporate Car Drivers", in Proceedings of the 2012 ACM Conference on Ubiquitous Computing, ACM, Pittsburgh, Pennsylvania. 2012. ACM: New York, NY.

[28] Vagg, C., C.J. Brace, D. Hari, S. Akehurst, J. Poxon, and L. Ash, "Development and Field Trial of a Driver Assistance System to Encourage Eco-Driving in Light Commercial Vehicle Fleets", IEEE Transactions on Intelligent Transportation Systems, 14(2), 2013, pp. 796805.

[29] van der Voort, M., M.S. Dougherty, and M. van Maarseveen, "A Prototype Fuel-Efficiency Support Tool", Transportation Research Part C: Emerging Technologies, 9(4), 2001, pp. 279-296. 